MAGNETOHYDRODYNAMICS Vol. 54 (2018), No. 1-2, pp. 11-14

DOI: $10.22364 / \mathrm{mhd} .54 .1-2.2$

\title{
EXCITATION AUTOWAVE SPREADING MODES IN THE NEAR-ELECTRODE LAYER OF MAGNETIC FLUID
}

\author{
N.V.Kandaurova ${ }^{1}$, V.V.Chekanov ${ }^{2}$, V.S.Chekanov ${ }^{1}$ \\ 1 Moscow Technological University (MIREA, Stavropol Branch), \\ 18 Kulakova pr., 355029 Stavropol, Russia \\ e-Mail: candaur18@yandex.ru \\ ${ }^{2}$ North-Caucasus Federal University, 1 Pushkin str., Stavropol, Russia
}

\begin{abstract}
Autowaves arise in various media of chemical, physical, biological origin. An important example of an excitable active medium in which autowave processes occur are biological tissues, including myocardium. The spreading of the nerve impulse in the cardiac muscle is autowave by nature and obeys the universal laws of autowave spreading. Violation of the autowave spreading modes leads to serious pathologies. Control of the arising wave with the help of external influences makes it possible to eliminate such pathology. These considerations determine the importance of autowave process investigations. In this paper, we investigate the modes of autowave excitation spreading which were observed in the near-electrode layer of magnetic fluid.
\end{abstract}

1. Experimental results and discussion. Experimental technique. It is known that near-electrode layers are formed in a magnetic fluid (MF) placed in a cell with transparent electrodes (a flat capacitor) in the electric field. They consist of close-packed magnetite particles covered with protective shells. This layer is an excitable medium in which an autowave process (AWP) was observed. Visualization of the AWP is based on the electro-optical effect occurring in a magnetic fluid exposed to an electric field: a layer of magnetite particles has a refractive index which is close in value to the refractive index of a transparent electrode (ITO). That is, the electrode-near-electrode layer structure is optically homogeneous. The thickness of the layer varies depending on the voltage applied to the electrodes in the cell. If the surface of the cell is illuminated with white light, it will be reflected from the borders of the glass-electrode and electrode + magnetite particles layer-magnetic fluid. The surface of the cell gets differently colored depending on the thickness of the electrode-magnetite particle layer structure. This phenomenon is known as the colors of thin membranes. The maximum of the reflected light spectrum shifts towards the long waves with an increase in near-electrode layer thickness.

2. "Fast" autowaves mode. A technique for the calculation of the nearelectrode layer thickness is presented in [1]. Depending on the cell voltage applied to the electrodes, the thickness of the layer varies up to $100 \mathrm{~nm}$. The specific resistance of this thickness layer is several orders of magnitude greater than the specific resistance of the liquid in the cell (the thickness of the liquid layer in the cell is $\sim 40 \mu \mathrm{m}$ ). Hence, the field strength at a steady-state current in a layer of such thickness also will be several orders of magnitude greater. At some critical voltage on the electrodes, the tension in the layer becomes $\sim 10^{7} \mathrm{~V} / \mathrm{m}$, it becomes conductive (Wien effect). Individual elements (ensembles of particles) acquire the same charge from the electrode and push away from it. A running wave is visible on the surface of the cell (Figs. 1). In color photographs, dark color corresponds to green, light color to orange and crimson. 


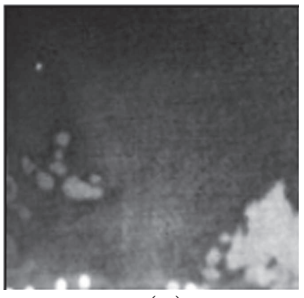

(a)

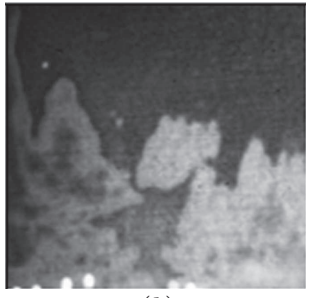

(b)

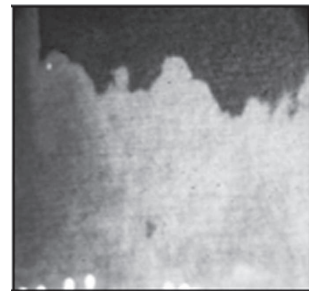

$(c)$

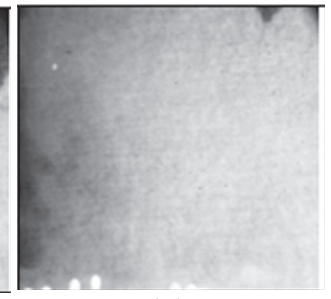

$(d)$

Fig. 1. "Fast" wave in the near-electrode layer of a cell with magnetic fluid.

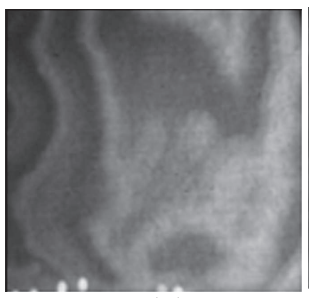

(a)

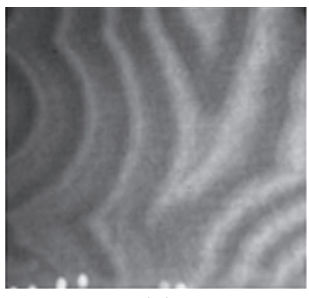

(b)

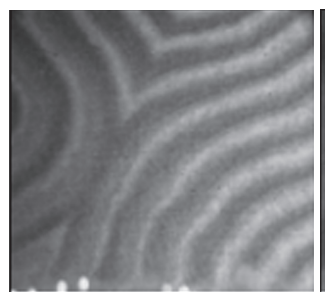

(c)

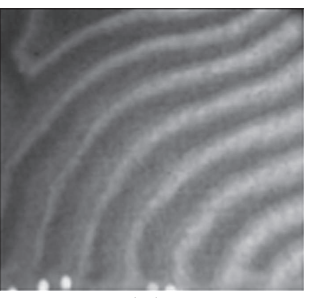

$(d)$

Fig. 2. Phase ("slow") autowaves in the near-electrode layer of a cell with magnetic fluid.

Note that complete information about the autowave structure evolution can be obtained by describing only the time evolution of the wave front position. This is the basis for the kinematic approach to the description of autowave structures [2].

Since the cell has dimensions of $3 \times 4 \mathrm{~cm}$, we can estimate the average velocity of the wave front motion. Approximately $0.25 \mathrm{~s}$ passes from the appearance of green color areas (Fig. 1a) before filling the entire surface of the cell with this color. Such a wave is called "fast", the velocity of its movement is about $16 \mathrm{~cm} / \mathrm{s}$. It looks like a flame spreading over the steppe by setting fire to different places. This is a well-known problem solved by Zeldovich and Frank-Kamenetsky.

3. "Slow" autowaves mode. Further, the layer elements (ensembles of particles) fall into the cell, break apart, lose the same charge as the electrode. They are oppositely charged in the cell and begin their movement to the electrode due to electro- and dipolar phoresis. Behind the first wave follows the second one, on the surface of the cell there appears a picture of the autowave process (Fig. 2). The waves move with a velocity of $\approx 1 \mathrm{~cm} / \mathrm{s}$. These are the so-called phase ("slow") autowaves. That is, the MF near-electrode layer is an excitable medium with a restoration.

\section{Relaxation self-oscillations. Modeling the work of the heart.}

It has been revealed in [3] that the resistance of a cell when a direct current passes through it increases several times as a result of the formation of electrode layers. In the same paper, it was shown that the capacitance associated with the passage of the current through the cell (polarization capacitance) was 3-4 orders of magnitude greater than the static capacity of the cell with the MF. Such increase in cell capacitance in the electric field, as well as the increase in resistance, can be associated only with the appearance of near-electrode layers. Since the thickness of the layers depends on the voltage on the electrode, the charge, the capacitance and the resistance measured in the discharge current also depend on the voltage on the electrodes. Thus, the MF cell is a variable capacitor.

If an additional variable resistance $R=0 \div 4 \mathrm{M} \Omega$ is connected in series, assuming the fact that the cell with a magnetic fluid (capacitor) has a large capacitance, 


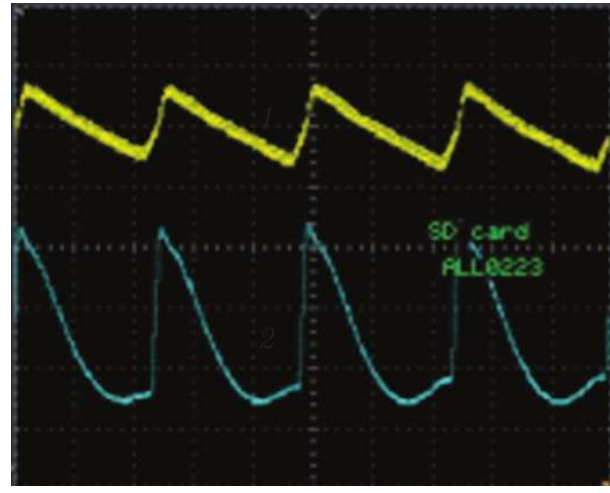

(a)

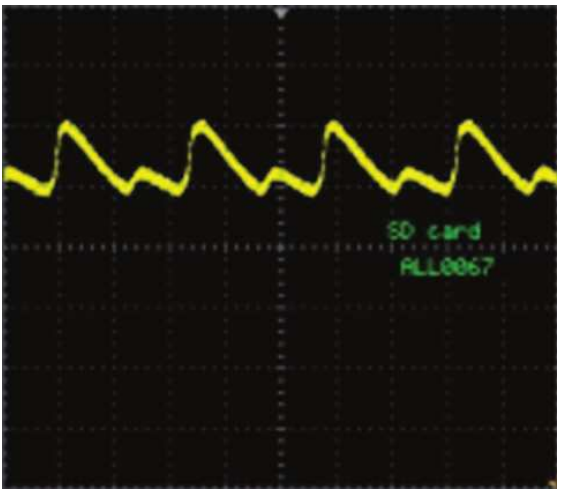

(b)

Fig. 3. (a) - oscillogram of the relaxation self oscillations of the accumulationdischarge type current in a cell with MF with no obstacle. Curve 1: current vs. time; curve 2: optical signal; (b) - oscillogram of relaxation oscillations of the current in a cell with MF with an obstacle.

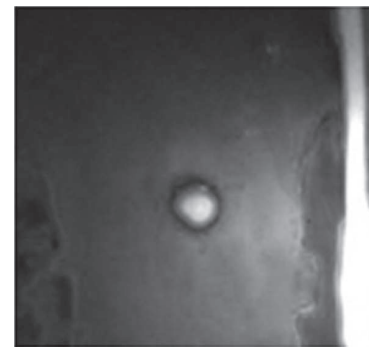

(a)

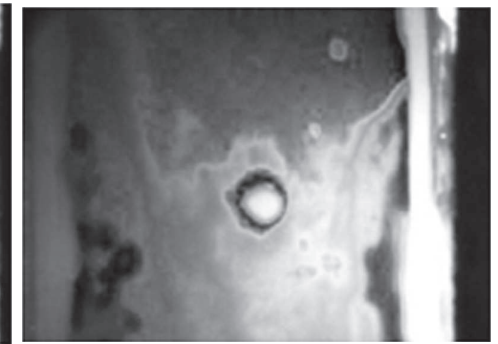

(b)

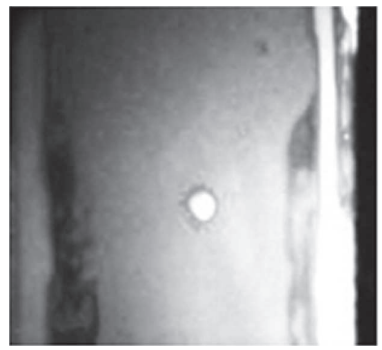

(c)

Fig. 4. Autowaves spreading in a medium with local inhomogeneity.

relaxation oscillations of the "accumulation-discharge" current appear in the nearelectrode layer (Fig. 3a, curve 1). The figure shows that the period of oscillation can be split into several sharply demarcated stages, corresponding to slow and rapid changes in the state of the system. The fast stage corresponds to the excitation time of the system, that is, to the discharge of the capacitor (via a shunt). The slow stage corresponds to the refractory period, i.e. to the formation of a layer, to the charge accumulation up to the instability threshold. Refractoriness is one of the most important properties of the excitable media, i.e. they need some time to restore their properties after pulse passing (capacitor discharge).

If the surface of the cell is illuminated with monochromatic light (laser), information on the thickness of the near-electrode layer can be obtained from the intensity of the reflected beam [1]. Curve 2 in Fig. $3 a$ is the optical response of the reflected beam $(\lambda=650 \mathrm{~nm})$. The maximum of the optical signal corresponds to the beginning of the near-electrode layer formation, i.e. the beginning of the capacitor (MF cell) charging. The green color of the cell surface is visible. The beginning of the discharge corresponds to the destruction of the layer, i.e. a sharp change in color of the cell surface from green to crimson. The charge time was $0.5 \mathrm{~s}$, the discharge time was $0.1 \mathrm{~s}$. It can be assumed that the observed oscillation relaxation process simulates the work of myocardium.

5. Spreading of autowaves in a medium with local inhomogeneity. Modelling of cardiac pathology. A local inhomogeneity was created in the near-electrode layer of the cell with a magnetic fluid, as shown in Fig. 4. The 


\section{N.V. Kandaurova, V.V.Chekanov, V.S. Chekanov}

oscillogram of the current, in this case, has the form illustrated in Fig. 3b. The stages of the autowave spreading are presented in Fig. 4 which shows that around the inhomogeneity there is an area, where oscillations occur with a phase different from the vibrations of the cell surface. The oscillogram in Fig. $3 b$ shows that the region around the local inhomogeneity has a refractory time $R_{1}$ which is different from the refractory time $R_{2}$ of the medium. Therefore, the wave front reaches the area with the refractory $R_{1}$ and breaks down. Therefore, arrhythmia arises in the oscillations between the entire surface of the cell and the region around the local inhomogeneity. If there is the inhomogeneity in the myocardium (muscle middle layer) of the heart, e.g., a heart attack, which means that there is no blood supply to some area, a similar rhythm transformation may occur in the cardiac muscle.

6. Conclusions. A layer of particles of magnetic fluid dispersed phase formed at the surface of a transparent electrode in the electric field is an excitable medium. The advantage of such medium is that an unlimited number of autowave cycles can be obtained, in contrast, for example, to the known BelousovZhabotinsky reaction, where the number of observed autowave cycles is limited by the reaction termination. Visualization of the autowave patterns is based on the electro-optical effect - electro-interference - arising in a magnetic fluid in an electric field. Various modes of the autowave spreading in excitable media have been investigated: fast and slow autowaves, relaxation self oscillations, autowaves in a medium with local inhomogeneity. The observed autowave processes can be used to model the processes taking place in the myocardium.

Acknowledgements. The presented study is an element of the basic part of the State Project No. 3.5385.2017/8.9/BCH "Experimental Research and Mathematical Modelling of the Interphase and Near-Surface Phenomena in a Thin Membrane of a Nanostructured Magnetic Fluid".

\section{References}

[1] V.V. Chekanov, N.V. Kandaurova, V.S. Chekanov. Journal of Nanoand Electronic Physics, vol. 8 (2016), no. 4, 040456.

[2] V.A. Davydov, V.S.Zykov, A.S. Mikhailov. Advances in Physical Sciences, vol. 161 (1991), no. 8, pp. 45-83.

[3] V.V. Chekanov, N.V. Kandaurova, V.S. Chekanov. Polarization capacitance and resistance of the near-electrode nanolayer of a magnetic fluid in an electric field. In: Proc. the 3rd International Scientific and Practical Conference "Physics and Technology of Nanomaterials and Structures (Kursk, May 23-25, 2017). 